\title{
On planar quantum dynamics of a magnetic dipole moment in the presence of electric and magnetic fields
}

\author{
Edilberto O. Silva ${ }^{\mathrm{a}}$ \\ Departamento de Física, Universidade Federal do Maranhão, Campus Universitário do Bacanga, 65085-580 São Luís, MA, Brazil
}

Received: 29 August 2014 / Accepted: 1 October 2014 / Published online: 15 October 2014

(C) The Author(s) 2014. This article is published with open access at Springerlink.com

\begin{abstract}
The planar quantum dynamics of a neutral particle with a magnetic dipole moment in the presence of electric and magnetic fields is considered. The criteria to establish the planar dynamics reveal that the resulting nonrelativistic Hamiltonian has a simplified expression without making approximations, and some terms have crucial importance for the system dynamics.
\end{abstract}

\section{Introduction}

The Aharonov-Bohm effect [1] has been an usual framework for demonstrating the importance of potentials in quantum mechanics. After its experimental verification $[2,3] \mathrm{sev}-$ eral other analog effects were being proposed along the last decades. For example, in Ref. [4], it was shown that a particle with a magnetic moment moving in an electric field acquires a quantum phase. This phase has been observed in a neutron interferometer [5] and in a neutral atomic Ramsey interferometer [6]. In Refs. [7,8] it was verified that a neutral particle with an electric dipole moment which moves in a magnetic field acquires a topological phase. The experimental confirmation of this phase was established in Ref. [9]. In Ref. [10] was proposed a unified and fully relativistic treatment of the interaction of the electric and magnetic dipole moments of a particle with the electromagnetic field. This study in essence reveals that a new force on dipoles is obtained using the non-Abelian nature of this interaction, and new experiments analogous to the Aharonov-Bohm effect to test this interaction are proposed. Since this interaction is a consequence of a nonminimal coupling, it is also interesting to analyze the consequences of this interaction in other contexts. For instance, it may be of interest to study scattering and bound states of neutral fermions in external electromagnetic fields and access other physical quantities such as energy bound states $[11,12]$ and scattering $[13,14]$. The dual results of the

a e-mail: edilbertoo@gmail.com magnetic dipole interaction for the electric dipole interaction for the system considered in Ref. [10] has been established in Ref. [15], where the phase shift in the interference of a magnetic or electric dipole due to the electromagnetic field is obtained relativistically and nonrelativistically.

In this work, we consider the same system as addressed in Ref. [15] but now assuming that the dynamics is purely planar, and we derive its equation of motion. This system is a generalization, for example, of that studied in Ref. [12], where only the effects of an electric field has been considered. An interesting feature of this system is that even in the absence of an electric field it admits bound and scattering states, which does not occur in the previous work. As an application, we consider the problem of a bound state for the case of a magnetic dipole moment interacting with electric and magnetic fields generated by an infinitely long charged solenoid, carrying a magnetic field. In our treatment, we consider the self-adjoint extension method [16], which is appropriate for addressing any system endowed with a singular Hamiltonian (due to localized field sources or quantum confinement) [17-26]. We determine the energy spectrum and wave functions by applying boundary conditions allowed by the system.

\section{The planar Pauli equation}

We begin with the Dirac equation in $(3+1)$ dimensions [15], which governs the nonrelativistic dynamics of a neutral particle that possesses a magnetic dipole moment, in the presence of electric and magnetic fields $(\hbar=c=1)$

$\left[i \gamma^{\mu} \partial_{\mu}-M+\frac{\mu}{2} \sigma^{\mu v} F_{\mu \nu}\right] \Psi=0$

where $\mu$ is the magnetic dipole moment, $F_{\mu \nu}$ is the electromagnetic tensor whose components are given by $\left(F^{0 i}, F^{i j}\right)=\left(-E^{i},-\varepsilon^{i j k} B^{k}\right)$, and $\left(\sigma^{0 j}, \sigma^{i j}\right)=\left(i \alpha^{j}\right.$, 
$\left.-\epsilon_{i j k} \Sigma^{k}\right)$, where $\Sigma^{k}$ is the spin operator, are the components of the operator $\sigma^{\mu \nu}=i\left[\gamma^{\mu}, \gamma^{\nu}\right] / 2$, which are given in terms of the Dirac matrices. With this notation, it is possible to show that the spin is coupled to the electromagnetic field tensor through the term

$\frac{1}{2} \sigma^{\mu \nu} F_{\mu \nu}=-\boldsymbol{\Sigma} \cdot \mathbf{B}+i \boldsymbol{\alpha} \cdot \mathbf{E}$,

where $\mathbf{E}$ and $\mathbf{B}$ are the electric and magnetic field strengths. This result is explicitly calculated in the following representation of the $\gamma$-matrices:

$$
\begin{aligned}
\gamma^{0} & =\left(\begin{array}{cc}
1 & 0 \\
0 & -1
\end{array}\right), \quad \boldsymbol{\gamma}=\left(\begin{array}{cc}
0 & \boldsymbol{\sigma} \\
-\boldsymbol{\sigma} & 0
\end{array}\right), \\
\boldsymbol{\alpha} & =\gamma^{0} \boldsymbol{\gamma}=\left(\begin{array}{ll}
0 & \boldsymbol{\sigma} \\
\boldsymbol{\sigma} & 0
\end{array}\right), \quad \boldsymbol{\Sigma}=\left(\begin{array}{ll}
\boldsymbol{\sigma} & 0 \\
0 & \boldsymbol{\sigma}
\end{array}\right) .
\end{aligned}
$$

with $\boldsymbol{\sigma}=\left(\sigma_{1}, \sigma_{1}, \sigma_{3}\right)$ being the Pauli matrices. Equation (1) can be written as

$\hat{H}_{D} \Psi=\mathscr{E} \Psi$,

where the operator

$$
\hat{H}_{D}=\beta M+\boldsymbol{\alpha} \cdot \mathbf{p}+\mu(-\beta \boldsymbol{\Sigma} \cdot \mathbf{B}+i \boldsymbol{\gamma} \cdot \mathbf{E})
$$

is the Dirac Hamiltonian. The nonrelativistic limit of Eq. (3) was established in Ref. [15], and the relevant equation is found to be

$\hat{H} \psi=E \psi$,

where $\psi$ is a two-component spinor, with

$$
\begin{aligned}
\hat{H}= & \frac{1}{2 M}[\mathbf{p}-(\boldsymbol{\mu} \times \mathbf{E})]^{2}-\frac{1}{2 M} \mu^{2} E^{2} \\
& +\frac{1}{2 M} \mu(\nabla \cdot \mathbf{E})-(\boldsymbol{\mu} \cdot \mathbf{B}),
\end{aligned}
$$

where $\boldsymbol{\mu}=\mu \boldsymbol{\sigma}$. Our goal is to analyze the physical implications of the Hamiltonian (6), when we assume that the system dynamics is now planar. This is established as follows. By detaching the third component of Eq. (6), we get

$$
\begin{aligned}
\hat{H}= & \frac{1}{2 M} \sum_{i=1}^{2}\left[\mathbf{p}_{i}-(\boldsymbol{\mu} \times \mathbf{E})_{i}\right]^{2}-\frac{1}{2 M} \mu^{2} E^{2} \\
& +\frac{1}{2 M}\left[\mathbf{p}_{3}-(\boldsymbol{\mu} \times \mathbf{E})_{3}\right]^{2} \\
& +\frac{1}{2 M} \sum_{i=1}^{2} \mu(\boldsymbol{\nabla} \cdot \mathbf{E})_{i}+\frac{1}{2 M} \mu(\boldsymbol{\nabla} \cdot \mathbf{E})_{3} \\
& -\sum_{i=1}^{2}(\boldsymbol{\mu} \cdot \mathbf{B})_{i}-(\boldsymbol{\mu} \cdot \mathbf{B})_{3}, \quad(i=1,2) .
\end{aligned}
$$

If we assume that the dynamics is planar, the above Hamiltonian provides us with an important result, namely, the $\left[\mathbf{p}_{3}-(\boldsymbol{\mu} \times \mathbf{E})_{3}\right]^{2}$ term leads exactly to the quantity $\mu^{2} E^{2} / 2 M$. The planar case is accessed by requiring that $p_{z}=z=0$ together with the imposition of the fields should not have a third direction. This question can also be understood when we look at the symmetry under $z$ translations, which allows us to access the solutions of the planar Dirac equation. This type of simplification is in fact manifest only when we assume that the particle moves in a plane. Thus, since $A^{\mu}=(\Phi, \mathbf{A})$, we write the electric and magnetic fields, respectively, as

$\mathbf{E}=-\hat{\mathbf{x}} \partial_{x} \Phi(x, y)-\hat{\mathbf{y}} \partial_{y} \Phi(x, y)$,

$\mathbf{B}=\hat{\mathbf{z}}\left(\partial_{x} A_{y}-\partial_{y} A_{x}\right)$.

The fields $\mathbf{E}$ and $\mathbf{B}$ above are now intrinsically twodimensional. Note that the square of Eq. (8) gives exactly $E_{1}^{2}+E_{2}^{2}$ for the planar case. Also, the restriction imposed on the potential A reveals that the $(\boldsymbol{\mu} \cdot \mathbf{B})_{i}$ term in Eq. (7) is now identically zero. Now, we can show that the quantity $(\boldsymbol{\mu} \times \mathbf{E})_{3}$ is given by $\mu\left(\sigma_{1} E_{2}-\sigma_{2} E_{1}\right)$, and the third term of Eq. (7) results in

$\left[\mathbf{p}_{3}-(\boldsymbol{\mu} \times \mathbf{E})_{3}\right]^{2} \rightarrow \mu^{2}\left(\sigma_{1} E_{2}-\sigma_{2} E_{1}\right)^{2}=\mu^{2} E^{2}$.

Thus, we now can write Eq. (7) as

$\hat{H}=\frac{1}{2 M} \sum_{i=1}^{2}\left\{\left[\mathbf{p}_{i}-(\boldsymbol{\mu} \times \mathbf{E})_{i}\right]^{2}+\mu(\boldsymbol{\nabla} \cdot \mathbf{E})_{i}\right\}-(\boldsymbol{\mu} \cdot \mathbf{B})_{3}$,

where the magnetic interaction term $(\boldsymbol{\mu} \cdot \mathbf{B})_{3}$ gives the only explicit dependence of the spin. In Ref. [15] it was assumed that the charge density $\rho=\nabla \cdot \mathbf{E}$ and also the $\mu^{2} E^{2} / 2 M$ term (for thermal neutrons) are negligible. In fact, this approximation can only be performed, if we are only interested in a study of the phase shift. However, if we want to study the dynamics of the system, such as the scattering and bound states problems, all terms of the equation of motion must be taken into account. This, for example, has been addressed by Hagen [27] to show that there is an exact equivalence between the $\mathrm{AB}$ and $\mathrm{AC}$ effects for spin- $1 / 2$ particles. In these effects, $\boldsymbol{\nabla} \cdot \mathbf{E}$ and $\boldsymbol{\nabla} \times \mathbf{A}$ being proportional to a delta function, such terms must now contribute to the dynamics of the system, and they cannot be neglected. For this reason, since we are dealing with a Aharonov-Bohm-like system for spin-1/2 particles, the $\mu(\boldsymbol{\nabla} \cdot \mathbf{E})_{i}$ and $(\boldsymbol{\mu} \cdot \mathbf{B})_{3}$ terms in Eq. (11), may not be negligible.

Let us clarify this issue. Consider an infinitely long solenoid, carrying a magnetic field $\mathbf{B}$, and with a charge density $\lambda$ distributed uniformly along the $z$-axis. The electric field 
and magnetic flux tube (in cylindrical coordinates) generated by this configuration are known to be

$\mathbf{E}=2 \lambda \frac{\hat{\mathbf{r}}}{r}, \nabla \cdot \mathbf{E}=2 \lambda \frac{\delta(r)}{r}$,

$\mathbf{B}=\boldsymbol{\nabla} \times \mathbf{A}=\phi \frac{\delta(r)}{r} \hat{\mathbf{z}}$

where $\phi$ is the magnetic flux inside the tube, and the vector potential in the Coulomb gauge is

$\mathbf{A}=\frac{\phi}{r} \hat{\varphi}$

We see that the fields $\mathbf{E}$ and $\mathbf{B}$ are proportional to a $\delta$ function. By using Eqs. (12) and (13), the Pauli equation (5) is now written as

$\hat{H} \psi=E \psi$

with

$\hat{H}=\frac{1}{2 M} \sum_{i=1}^{2}\left(\mathbf{p}_{i}-\phi_{E} \sigma_{z} \frac{\hat{\varphi}}{r}\right)^{2}+\left(\phi_{E}-\phi_{B} \sigma_{z}\right) \frac{\delta(r)}{r}$,

where $\phi_{E}=2 \mu \lambda$ and $\phi_{B}=2 M \mu \phi$. From Eq. (15), we can see that $\psi$ is an eigenfunction of $\sigma_{z}$, whose eigenvalues are designated by $s= \pm 1$, that is, $\sigma_{z} \psi= \pm \psi=s \psi$. Thus, since $\hat{H}$ commutes with the operators $\hat{J}_{z}=-i \partial_{\varphi}+\sigma_{z} / 2$, where $\hat{J}_{z}$ is the total angular momentum operator in the $z$-direction, we seek solutions of the form

$\psi(r, \varphi)=\left[\begin{array}{c}f_{m}(r) \mathrm{e}^{i m \varphi} \\ g_{m}(r) \mathrm{e}^{i(m+1) \varphi}\end{array}\right]$

with $m+1 / 2= \pm 1 / 2, \pm 3 / 2, \ldots,(m \in \mathbb{Z})$. Inserting (17) into Eq. (15), we can extract the radial equation for $f_{m}(r)$,

$H f_{m}(r)=k^{2} f_{m}(r)$,

where

$H=H_{0}+\left(\phi_{E}-s \phi_{B}\right) \frac{\delta(r)}{r}$

and

$H_{0}=-\frac{\mathrm{d}^{2}}{\mathrm{~d} r^{2}}-\frac{1}{r} \frac{\mathrm{d}}{\mathrm{d} r}+\frac{\left(m-s \phi_{E}\right)^{2}}{r^{2}}$.

Note that, even in the absence of an electric field, bound and scattering states are possible. This does not occur, for example, in the system studied in Ref. [12], where the particle interacts only with an electric field. Moreover, if a magnetic field is present, the physical system changes completely. We will see later that this fact directly influences the expression for the self-adjoint extension parameter and, hence, on the boundary conditions allowed by the operator $H_{0}$. In other words, this has direct implications on the dynamics of the system. This can be seen more easily by studying the signal of $\phi_{E}-s \phi_{B}$ in Eq. (19), where several possible combinations of $\phi_{E}, \phi_{B}$ and $s$ give us the possibilities for the existence of bound and scattering states. As a result of these combinations, we have

$\phi_{E}-s \phi_{B}<0$, scattering and bound states,

$\phi_{E}-s \phi_{B}>0$, scattering states.

The case $\phi_{E}=s \phi_{B}$ is not of interest here because it cancels the term that explicitly depends on the spin.

\section{Physical regularization and the bound states problem}

In this section, we study the dynamics of the system in the whole space, including the $r=0$ region. We consider the problem of bound states. To this end, we use the self-adjoint extension method in the treatment. As is well known, if the Hamiltonian has a singularity point, as is the case of the Hamiltonian in Eq. (19), we must verify that it is selfadjoint in the region of interest. Even though $H_{0}^{\dagger}=H_{0}$, their domains could be different. This is the crucial point in our study. The operator $H_{0}$, with domain $\mathscr{D}\left(H_{0}\right)$, is self-adjoint if $\mathscr{D}\left(H_{0}^{\dagger}\right)=\mathscr{D}\left(H_{0}\right)$ and $H_{0}^{\dagger}=H_{0}$. However, for this to be established, we must find the deficiency subspaces,

$N_{+}=\left\{\psi \in \mathscr{D}\left(H_{0}^{\dagger}\right), H_{0}^{\dagger} \psi=z_{+} \psi, \operatorname{Im} z_{+}>0\right\}$,
$N_{-}=\left\{\psi \in \mathscr{D}\left(H_{0}^{\dagger}\right), H_{0}^{\dagger} \psi=z_{-} \psi, \operatorname{Im} z_{-}<0\right\}$,

with dimensions $n_{+}$and $n_{-}$, respectively, called deficiency indices of $H_{0}$ [16]. We also know of this theory that a necessary and sufficient condition for $H_{0}$ being essentially selfadjoint is that its deficiency indices $n_{+}=n_{-}=0$. On the other hand, if $n_{+}=n_{-} \geq 1$ the operator $H_{0}$ has an infinite number of self-adjoint extensions parametrized by the unitary operators $U: N_{+} \rightarrow N_{-}$. With these ideas in mind, we now decompose the Hilbert space $\mathscr{H}=L^{2}\left(\mathbb{R}^{2}\right)$ with respect to the angular momentum $\mathscr{H}=\mathscr{H}_{r} \otimes \mathscr{H}_{\varphi}$, where $\mathscr{H}_{r}=L^{2}\left(\mathbb{R}^{+}, r \mathrm{~d} r\right)$ and $\mathscr{H}_{\varphi}=L^{2}\left(S^{1}, \mathrm{~d} \varphi\right)$, with $S^{1}$ denoting the unit sphere in $\mathbb{R}^{2}$. The operator $-\partial_{\varphi}^{2}$ is known to be essentially self-adjoint in $L^{2}\left(S^{1}, \mathrm{~d} \varphi\right)$. By using the unitary operator [18]

$V: L^{2}\left(\mathbb{R}^{+}, r \mathrm{~d} r\right) \rightarrow L^{2}\left(\mathbb{R}^{+}, \mathrm{d} r\right)$

given by

$(V Q)(r)=r^{1 / 2} Q(r)$, 
the operator $H_{0}$ reads

$H_{0}^{\prime}=V H_{0} V^{-1}=-\frac{1}{2 M}\left\{\frac{\mathrm{d}^{2}}{\mathrm{~d} r^{2}}+\frac{1}{r^{2}}\left[\left(m-s \phi_{E}\right)^{2}-\frac{1}{4}\right]\right\}$,

which is essentially self-adjoint for $\left(m-s \phi_{E}\right) \geq 1$, while for $\left(m-s \phi_{E}\right)<1$, it admits a one-parameter family of self-adjoint extensions [16]. To characterize this family, we follow the recipe based on the boundary conditions given in Ref. [28]. Basically, the boundary condition is a match of the logarithmic derivatives of the zero-energy solutions for Eq. (18) and the solutions for the problem $H_{0}$ plus the self-adjoint extension. Then, following [28], we temporarily forget the $\delta$ function potential and find the boundary conditions allowed for $H_{0}$. Next, we substitute the problem in Eq. (18) by

$H_{0} f_{\zeta}(r)=k^{2} f_{\zeta}(r)$,

plus self-adjoint extensions. Here, $f_{\zeta}$ is labeled by the parameter $\zeta$ of the self-adjoint extension, which is related to the behavior of the wave function at the origin. In order for the $H_{0}$ to be a self-adjoint operator in $\mathscr{H}_{r}$, its domain of definition has to be extended by the deficiency subspace, which is spanned by the solutions of the eigenvalue equation

$H_{0}^{\dagger} f_{ \pm}(r)= \pm i k_{0}^{2} f_{ \pm}(r)$

where $k_{0}^{2} \in \mathbb{R}$ is introduced for dimensional reasons. Since $H_{0}^{\dagger}=H_{0}$, the only square integrable functions which are solutions of Eq. (29) are the modified Bessel functions of the second kind,

$f_{ \pm}(r)=K_{m-s \phi_{E}}\left(\sqrt{\mp i} k_{0} r\right)$,

with $\operatorname{Im} \sqrt{ \pm i}>0$. By studying Eq. (30), we verify that it is square integrable only in the range $m-s \phi_{E} \in(-1,1)$. In this interval, nevertheless, the Hamiltonian (20) is not self-adjoint. The dimension of such deficiency subspace is $\left(n_{+}, n_{-}\right)=(1,1)$. So, we have two situations for $m-s \phi_{E}$, i.e.,

$-1<m-s \phi_{E}<0$,

$0<m-s \phi_{E}<1$.

To address both cases of Eq. (31), we write Eq. (30) as

$f_{ \pm}(r)=K_{\left|m-s \phi_{E}\right|}\left(\sqrt{\mp i} k_{0} r\right)$.

Equation (32) allows us to identify the domain of $H_{0}^{\dagger}$ as

$\mathscr{D}\left(H_{0}^{\dagger}\right)=\mathscr{D}\left(H_{0}\right) \oplus N_{+} \oplus N_{-}$.
So, to extend the domain $\mathscr{D}\left(H_{0}\right)$ and make it equal to $\mathscr{D}\left(H_{0}^{\dagger}\right)$ and therefore to turn $H_{0}$ self-adjoint, we get

$\mathscr{D}\left(H_{0, \varsigma}\right)=\mathscr{D}\left(H_{0}^{\dagger}\right)=\mathscr{D}\left(H_{0}\right) \oplus N_{+} \oplus N_{-}$.

Equation (34) establishes the following result. For each value of $\zeta$, we have a possible domain for $\mathscr{D}\left(H_{0, \zeta}\right)$, but the physical parameters are entailed of the problem that will select a particular value of it. The Hilbert space is now specified by [16]

$$
\begin{aligned}
f_{\zeta}(r)= & f_{m}(r)+C\left[K_{\left|m-s \phi_{E}\right|}\left(\sqrt{-i} k_{0} r\right)\right. \\
& \left.+\mathrm{e}^{i \zeta} K_{\left|m-s \phi_{E}\right|}\left(\sqrt{i} k_{0} r\right)\right]
\end{aligned}
$$

where $f_{m}(r)$, with $f_{m}(0)=\dot{f}_{m}(0)=0(\dot{f} \equiv \mathrm{d} f / \mathrm{d} r)$ is the regular wave function and the parameter $\zeta \in[0,2 \pi)$ represents a choice for the boundary condition. For each $\zeta$, we have a possible domain for $H_{0}$ and the physical situation is the factor that will determine the value of $\zeta$ [20,29-34]. Thus, to find a fitting for $\zeta$ compatible with the physical situation, a physically motivated form for the magnetic field is preferable for the regularization of the $\delta$-function. This is accomplished by replacing (14) with [35]

$e \mathbf{A}= \begin{cases}\frac{\phi}{r} \hat{\varphi}, & r>a \\ 0, & r<a .\end{cases}$

With this modification, the delta function in Eq. (19) is now regularized as $\delta(r-a) / a$. A remarkable feature of this regularization is that, although the functional structure of $\delta(r) / r$ and $\delta(r-a) / a$ are quite different, we are free to use any form of potential once the specific details of the regularization model can be shown to be irrelevant provided that only the contribution is independent of angle and has no $\delta$-function contribution at the origin [35]. It should also be mentioned that the $\delta(r-a) / a$ potential is one-dimensional and well defined, contrary to the two-dimensional $\delta(r) / r$.

Now, we are in a position to determine a fitting value for $\zeta$. To do so, we consider the zero-energy solutions for $f_{0}(r)$ with the regularization, and for $f_{\zeta, 0}(r)$ without the $\delta$ function, respectively, i.e.,

$$
\begin{aligned}
& \left\{-\frac{1}{r} \frac{\mathrm{d}}{\mathrm{d} r}\left(r \frac{\mathrm{d}}{\mathrm{d} r}\right)+\frac{\left(m-s \phi_{E}\right)^{2}}{r^{2}}\right. \\
& \left.+\left(\phi_{E}-s \phi_{B}\right) \frac{\delta(r-a)}{a}\right\} f_{0}(r)=0,
\end{aligned}
$$

$$
\left\{-\frac{1}{r} \frac{\mathrm{d}}{\mathrm{d} r}\left(r \frac{\mathrm{d}}{\mathrm{d} r}\right)+\frac{\left(m-s \phi_{E}\right)^{2}}{r^{2}}\right\} f_{\zeta, 0}(r)=0 .
$$


The value of $\zeta$ is determined by the boundary condition

$\left.\lim _{a \rightarrow 0^{+}} a \frac{\dot{f}_{0}(r)}{f_{0}(r)}\right|_{r=a}=\left.\lim _{a \rightarrow 0^{+}} a \frac{\dot{f}_{\zeta, 0}(r)}{f_{\zeta, 0}(r)}\right|_{r=a}$.

By integrating Eq. (37) from 0 to $a$ and noting that the behavior of $f_{0}$ as $a \rightarrow 0$ is $f_{0} \sim r^{\left|m-s \phi_{E}\right|}$, the left-hand side of Eq. (39) is found to be

$\left.\lim _{a \rightarrow 0^{+}} a \frac{\dot{f_{0}}(r)}{f_{0}(r)}\right|_{r=a}=\phi_{E}-s \phi_{B}$.

To calculate the right-hand side of Eq. (39), we need to use the asymptotic behavior for $K_{v}(z)$ in the limit $z \rightarrow 0$, given by

$K_{v}(z) \sim \frac{\pi}{2 \sin (\pi v)}\left[\frac{z^{-v}}{2^{-v} \Gamma(1-v)}-\frac{z^{v}}{2^{v} \Gamma(1+v)}\right]$.

The substitution of Eq. (41) into Eq. (35) leads to

$\left.\lim _{a \rightarrow 0^{+}} a \frac{\dot{f}_{\zeta, 0}(r)}{f_{\zeta, 0}(r)}\right|_{r=a}=\left.\lim _{a \rightarrow 0^{+}} \frac{\dot{W}_{\zeta}(r)}{W_{\zeta}(r)}\right|_{r=a}$,

with

$$
\begin{gathered}
W_{\zeta}(r)=\left[\frac{\left(\sqrt{-i} k_{0} r\right)^{-\left|m-s \phi_{E}\right|}}{2^{-\left|m-s \phi_{E}\right|} \Gamma^{(-)}}-\frac{\left(\sqrt{-i} k_{0} r\right)^{\left|m-s \phi_{E}\right|}}{2^{\left|m-s \phi_{E}\right|} \Gamma^{(+)}}\right] \\
+\mathrm{e}^{i \zeta}\left[\frac{\left(\sqrt{i} k_{0} r\right)^{-\left|m-s \phi_{E}\right|}}{2^{-\left|m-s \phi_{E}\right|} \Gamma^{(-)}} \frac{\left(\sqrt{i} k_{0} r\right)^{\left|m-s \phi_{E}\right|}}{2^{\left|m-s \phi_{E}\right|} \Gamma^{(+)}}\right]
\end{gathered}
$$

and $\Gamma^{( \pm)}=\Gamma\left(1 \pm\left|m-s \phi_{E}\right|\right)$ was defined for purposes of simplification. Inserting (40) and (42) in (39), we obtain

$\left.\lim _{a \rightarrow 0^{+}} \frac{\dot{W}_{\zeta}(r)}{W_{\zeta}(r)}\right|_{r=a}=\phi_{E}-s \phi_{B}$,

which gives us the parameter $\zeta$ in terms of the physics of the problem, i.e., the correct behavior of the wave functions when $r \rightarrow 0$.

As promised above, let us now derive determine the bound states for $H_{0}$. In order for a system to have a bound state, its energy must be negative, so that in Eq. (28), $k$ is a pure imaginary quantity, i.e., $k=i \kappa$, with $\kappa=\sqrt{-2 M E}$, where $E<0$ is the bound state energy. Then, with the substitution $k \rightarrow i \kappa$, we have

$$
\left\{\frac{1}{r} \frac{\mathrm{d}}{\mathrm{d} r}\left(r \frac{\mathrm{d}}{\mathrm{d} r}\right)-\left[\frac{\left(m-s \phi_{E}\right)^{2}}{r^{2}}+\kappa^{2}\right]\right\} f_{\zeta}(r)=0,
$$

The above equation is the modified Bessel equation whose general solution is given by

$f_{\zeta}(r)=K_{\left|m-s \phi_{E}\right|}(r \sqrt{-2 M E})$.

Since these solutions belong to $\mathscr{D}\left(H_{\zeta, 0}\right)$, it is of the form (35) for some $\zeta$ selected from the physics of the problem. So, we substitute (46) into (35) and use (41) to calculate the left-hand side of Eq. (39). After these manipulations, we find the relation

$$
\begin{aligned}
& \frac{\left|m-s \phi_{E}\right|\left[a^{2\left|m-s \phi_{E}\right|} \Gamma^{(-)}\left(-M E_{b}\right)^{\left|m-s \phi_{E}\right|}+2^{\left|m-s \phi_{E}\right|} \Gamma^{(+)}\right]}{a^{2\left|m-s \phi_{E}\right|} \Gamma^{(-)}\left(-M E_{b}\right)^{\left|m-s \phi_{E}\right|}-2^{\left|m-s \phi_{E}\right|} \Gamma^{(+)}} \\
& \quad=\phi_{E}-s \phi_{B} .
\end{aligned}
$$

Solving the above equation for $E$, we find the energy spectrum

$$
\begin{aligned}
E & =-\frac{2}{M a^{2}} \\
& \times\left[\left(\frac{\phi_{E}-s \phi_{B}+\left|m-s \phi_{E}\right|}{\phi_{E}-s \phi_{B}-\left|m-s \phi_{E}\right|}\right) \frac{\Gamma\left(1+\left|m-s \phi_{E}\right|\right)}{\Gamma\left(1-\left|m-s \phi_{E}\right|\right)}\right]^{\frac{1}{\left|m-s \phi_{E}\right|} .}
\end{aligned}
$$

Notice that there is no arbitrary parameter in the above equation. Also, to ensure that the energy is a real quantity, we must establish that

$$
\left(\frac{\phi_{E}-s \phi_{B}+\left|m-s \phi_{E}\right|}{\phi_{E}-s \phi_{B}-\left|m-s \phi_{E}\right|}\right) \frac{\Gamma\left(1+\left|m-s \phi_{E}\right|\right)}{\Gamma\left(1-\left|m-s \phi_{E}\right|\right)}>0 .
$$

This inequality is satisfied if

$\left|\phi_{E}-s \phi_{B}\right| \geq\left|m-s \phi_{E}\right|$.

Because of the condition that $\left|m-s \phi_{E}\right|<1$, it is sufficient to consider $\left|\phi_{E}-s \phi_{B}\right| \geq 1$. A necessary condition for a $\delta$-function to generate an attractive potential, which is able to support bound states, is that the coupling constant $\left(\phi_{E}-s \phi_{B}\right)$ must be negative. Thus, the existence of bound states requires

$\phi_{E}-s \phi_{B} \leq-1$.

Thus, it seems that we must have

$s \phi_{B}>\phi_{E}$,

in such way that the flux and the spin must be parallel, and consequently, a minimum value for $\left|\phi_{B}\right|$ and $\left|\phi_{E}\right|$ is established.

\section{Conclusions}

We have analyzed the planar quantum dynamics of a magnetic dipole moment in the presence of electric and magnetic fields. We have shown that the initial Hamiltonian system 
(Eq. 6) reduces to a planar form (Eq. 11) without making any approximations. As an application, we have considered the bound state problem for the case of a magnetic dipole moment interacting with electric and magnetic fields generated by an infinitely long solenoid, carrying a magnetic field, and with a charge density distributed uniformly about it along the $z$-axis. The self-adjoint extension approach was used to determine the bound states of the particle in terms of the physics of the problem, in a very consistent way and without any arbitrary parameter.

Acknowledgments The author would like to thank R. Casana and F. M. Andrade for the critical reading of the manuscript and for helpful discussions. This work was supported by CNPq (Grants No. 482015/2013-6 (Universal), No.306068/2013-3 (PQ)) and FAPEMA (Grant No. 00845/13 (Universal)).

Open Access This article is distributed under the terms of the Creative Commons Attribution License which permits any use, distribution, and reproduction in any medium, provided the original author(s) and the source are credited.

Funded by $\mathrm{SCOAP}^{3}$ / License Version CC BY 4.0.

\section{References}

1. Y. Aharonov, D. Bohm, Phys. Rev. 115(3), 485 (1959). doi:10. 1103/PhysRev.115.485

2. R.G. Chambers, Phys. Rev. Lett. 5, 3 (1960). doi:10.1103/ PhysRevLett.5.3

3. M. Peshkin, H.J. Lipkin, Phys. Rev. Lett. 74(15), 2847 (1995). doi:10.1103/PhysRevLett.74.2847

4. Y. Aharonov, A. Casher, Phys. Rev. Lett. 53(4), 319 (1984). doi:10. 1103/PhysRevLett.53.319

5. A. Cimmino, G.I. Opat, A.G. Klein, H. Kaiser, S.A. Werner, M. Arif, R. Clothier, Phys. Rev. Lett. 63, 380 (1989). doi:10.1103/ PhysRevLett.63.380

6. K. Sangster, E.A. Hinds, S.M. Barnett, E. Riis, Phys. Rev. Lett. 71, 3641 (1993). doi:10.1103/PhysRevLett.71.3641

7. X.G. He, B.H.J. McKellar, Phys. Rev. A 47, 3424 (1993). doi:10. 1103/PhysRevA.47.3424

8. M. Wilkens, Phys. Rev. Lett. 72, 5 (1994). doi:10.1103/ PhysRevLett.72.5

9. H. Wei, R. Han, X. Wei, Phys. Rev. Lett. 75, 2071 (1995). doi:10. 1103/PhysRevLett.75.2071

10. J. Anandan, Phys. Rev. Lett. 85, 1354 (2000). doi:10.1103/ PhysRevLett.85.1354
11. B. Roy, J. Phys. A: Math. Gen. 26(20), 5631 (1993). doi:10.1088/ 0305-4470/26/20/043

12. E.O. Silva, F.M. Andrade, C. Filgueiras, H. Belich, Eur. Phys. J. C 73(4), 2402 (2013). doi:10.1140/epjc/s10052-013-2402-1

13. Q.G. Lin, Phys. Rev. A 81(1), 012710 (2010). doi:10.1103/ PhysRevA.81.012710

14. Q.G. Lin, Phys. Rev. A 72(4), 042103 (2005). doi:10.1103/ PhysRevA.72.042103

15. J. Anandan, Phys. Lett. A 138(8), 347 (1989). doi:10.1016/ 0375-9601(89)90828-1

16. M. Reed, B. Simon, Methods of Modern Mathematical Physics. II. Fourier Analysis, Self-Adjointness (Academic Press, New York, 1975)

17. Y.N. Demkov, V.N. Ostrovskii, Zero-Range Potentials and their Applications in Atomic Physics (Plenum, New York, 1988)

18. S. Albeverio, F. Gesztesy, R. Høegh-Krohn, H. Holden, Solvable Models in Quantum Mechanics (Springer, Berlin, 1988)

19. F.M. Andrade, E.O. Silva, Phys. Lett. B 719(4-5), 467 (2013). doi:10.1016/j.physletb.2013.01.062

20. Ph. de Sousa Gerbert, Phys. Rev. D 40(4), 1346 (1989). doi:10. 1103/PhysRevD.40.1346

21. R. Adami, A. Teta, Lett. Math. Phys. 43(1), 43 (1998). doi:10.1023/ A:1007330512611

22. M.H. Al-Hashimi, U.J. Wiese, Ann. Phys. (NY) 327(11), 2742 (2012). doi:10.1016/j.aop.2012.06.006

23. V.R. Khalilov, Eur. Phys. J. C 73(8), 2548 (2013). doi:10.1140/ epjc/s10052-013-2548-x

24. V.R. Khalilov, Eur. Phys. J. C 74(1), 2708 (2014). doi:10.1140/ epjc/s10052-013-2708-z

25. V.R. Khalilov, Theor. Math. Phys. 163(1), 511 (2010). doi:10.1007/ s11232-010-0039-x

26. V.R. Khalilov, K.E. Lee, I.V. Mamsurov, Mod. Phys. Lett. A 27(05), 1250027 (2012). doi:10.1142/S0217732312500277

27. C.R. Hagen, Phys. Rev. Lett. 64(20), 2347 (1990). doi:10.1103/ PhysRevLett.64.2347

28. B.S. Kay, U.M. Studer, Commun. Math. Phys. 139(1), 103 (1991). doi:10.1007/BF02102731

29. C. Filgueiras, E.O. Silva, W. Oliveira, F. Moraes, Ann. Phys. (NY) 325(11), 2529 (2010). doi:10.1016/j.aop.2010.05.012

30. C. Filgueiras, F. Moraes, Ann. Phys. (NY) 323(12), 3150 (2008). doi:10.1016/j.aop.2008.08.002

31. C. Filgueiras, E.O. Silva, F.M. Andrade, J. Math. Phys. 53(12), 122106 (2012). doi: $10.1063 / 1.4770048$

32. F.M. Andrade, E.O. Silva, M. Pereira, Ann. Phys. (N.Y.) 339(0), 510 (2013). doi:10.1016/j.aop.2013.10.001

33. F.M. Andrade, E.O. Silva, M. Pereira, Phys. Rev. D 85(4), 041701(R) (2012). doi:10.1103/PhysRevD.85.041701

34. E.O. Silva, F.M. Andrade, Europhys. Lett. 101(5), 51005 (2013). doi:10.1209/0295-5075/101/51005

35. C.R. Hagen, Phys. Rev. Lett. 64(5), 503 (1990). doi:10.1103/ PhysRevLett.64.503 